\title{
Childhood Aleppo boil
}

\section{Pablo Fernández-Crehuet MD PhD, Ricardo Ruiz-Villaverde MD PhD}

Competing interests: None declared.

This article has been peer reviewed.

The authors have obtained patient consent.

Affiliations: Department of Dermatology (FernándezCrehuet), Hospital Alto Guadalquivir, Andújar, Jaén, Spain; Department of Dermatology (Ruiz-

Villaverde), Complejo

Hospitalario de Jaén, Jaén, Jaén, Spain

Correspondence to: Pablo Fernández-Crehuet, pablocrehuet@hotmail.com

CMAJ 2014. DOI:10.1503 /cmaj.140108
A seven-year-old girl presented with an asymptomatic papular skin lesion with central serous crust on her left cheek that had appeared two weeks earlier (Figure 1A). She had no history of an insect bite on her cheek or previous trauma. Polarized noncontact dermoscopy showed features consistent with cutaneous leishmaniasis (Figure 1B). Results of a potassium hydroxide examination, mycological culture and serologic test for Borrelia were negative. A polymerase chain reaction assay detected Leishmania donovani complex. An ointment containing paromomycin sulfate $15 \%$ and methylbenzethonium chloride $12 \%$ was applied nightly under occlusive dressing for 14 days, and the lesion resolved.

Old World cutaneous leishmaniasis is an endemic infection in the Mediterranean area transmitted by phlebotomine sandflies, with an incidence rate of 3.7-6.8 cases per 1000 population per year. ${ }^{1,2}$ The hallmark manifestation is the Aleppo boil, which usually develops within several weeks or months after the exposure and typically affects children. ${ }^{3}$ The differential diagnosis includes tinea incognito (which may be masked by topical corticosteroid use), acute prurigo and tache noir (as the initial clinical feature of Mediterranean spotted fever).

Polymerase chain reaction assay is the most sensitive test to diagnose Old World cutaneous leishmaniasis. Differing features will be seen on skin biopsy, depending on the phase of the lesion. Dermoscopy has substantially improved the diagnosis of this condition, as in our patient, who had typical features of Old World cutaneous leishmaniasis on dermoscopy. The main dermoscopic features of the condition include generalized erythema in $100 \%$ of cases, hyperkeratosis with or without central ulceration, and two recently identified patterns ("yellow tears" in 53\% of cases and a "white starburst-like pattern" in $38 \%$ of cases). ${ }^{3}$

There is no international gold standard for the optimal management of Old World cutaneous leishmaniasis, particularly because different Leishmania species differ in virulence. However, clinical guidelines are available for managing different clinical scenarios. ${ }^{2}$ The treatment approach for chil-

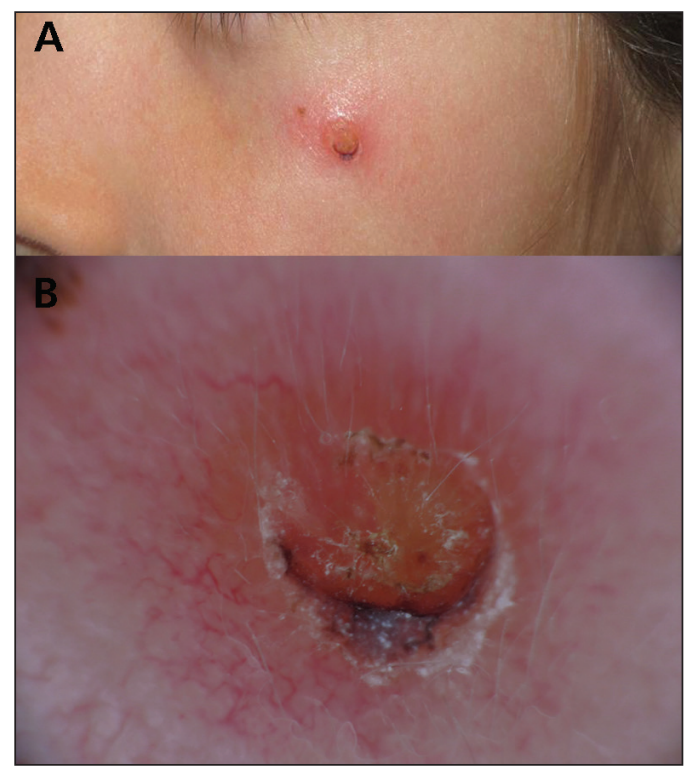

Figure 1: (A) Erythematous papule with serous central crust on the left cheek of a seven-year-old girl. (B) Polarized light dermoscopy showing basal erythema, central ulceration and hyperkeratosis with peripheral arborizing telangiectasia and white starburst-like pattern, consistent with Old World cutaneous leishmaniasis.

dren is complicated. Intralesional pentavalent antimonials often require several injections and may cause local or systemic toxicity. Other options include cryotherapy or photodynamic therapy, which are painful. The application of paromomycin sulfate $15 \%$ and methylbenzethonium chloride $12 \%$ twice daily for 20 days is an interesting therapeutic alternative, with cure rates between $77 \%$ and 94\%. ${ }^{4}$ To minimize the irritant effect and guarantee therapeutic compliance, occlusive treatment nightly for 14 days has been used with excellent response. ${ }^{1}$

\section{References}

1. García-Almagro D. Cutaneous leishmaniasis. Actas Dermosifiliogr 2005;96:1-24.

2. Goto H, Lindoso JA. Current diagnosis and treatment of cutaneous and mucocutaneous leishmaniasis. Expert Rev Anti Infect Ther 2010;8:419-33.

3. Llambrich A, Zaballos P, Terrasa F, et al. Dermoscopy of cutaneous leishmaniasis. Br J Dermatol 2009;160:756-61.

4. Monge-Maillo B, López-Vélez R. Therapeutic options for old world cutaneous leishmaniasis and new world cutaneous and mucocutaneous leishmaniasis. Drugs 2013;73:1889-920. 